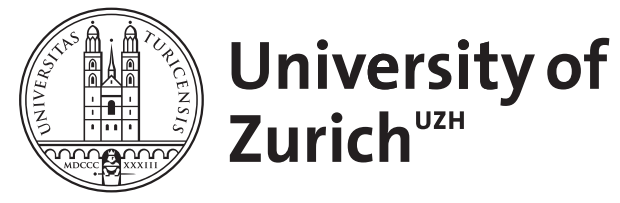

\title{
Muscle contraction mechanism
}

Schaub, M C ; Koch, D

DOI: https://doi.org/10.1016/s0008-6363(96)88608-1

Posted at the Zurich Open Repository and Archive, University of Zurich ZORA URL: https://doi.org/10.5167/uzh-155203

Journal Article

Published Version

Originally published at:

Schaub, M C; Koch, D (1995). Muscle contraction mechanism. Cardiovascular Research, 29(6):747-757. DOI: https://doi.org/10.1016/s0008-6363(96)88608-1 


\section{Commentaries}

\section{Muscle contraction mechanism}

\section{From Professor Sir Andrew Huxley}

$\mathrm{I}$ have been asked by the Editors of Cardiovascular Research to comment on the article by $\mathrm{G} \mathrm{H}$ Pollack in this issue. I will confine my remarks to aspects of the problem in which I have been personally involved.

In a general way, I agree with Pollack that there is a danger that an attractive theory may gain such wide acceptance that other possibilities do not get the consideration that they deserve, and I have said so in print more than once. On the other hand, I do not consider that Pollack's article is a useful contribution to the present debate.

In the first place, Pollack confuses the issue by failing to distinguish between the "swinging cross bridge theory" and the broader class of "independent force generator theories" in which contributions to the total force between thick and thin filaments are provided by identical force generators, which may or may not be swinging cross bridges, uniformly distributed through each overlap zone. This theory was suggested ${ }^{1}$ by the observation of Ramsey and Street $^{2}$ that active tension declines linearly with extension beyond the optimum length, implying that tension may be proportional to filament overlap.

Pollack's first objection to what he ambiguously calls the "cross bridge theory" is that there have been many reports that, at long lengths, an isolated frog fibre gives much more tension than expected from the amount of overlap of thin filaments with cross bridges on the thick filaments. Most of these reports are by Pollack and his coworkers. As far as I know, all such reports are based on measurements of tension made with the tendon ends of the fibre held stationary, and such mcasurcments had been shown by Peachey and myself in $1961^{3}$ to be highly misleading because the end regions of the fibre do not extend as much as the central region when the fibre is stretched: the end regions have more overlap and therefore generate more tension, which the central region is able to hold because of the well known resistance of active muscle to stretch. ${ }^{4}$ We also showed that, under isotonic conditions, shortening occurred only in parts of the fibre where the sarcomere length was such that there was overlap between thick and thin filaments. When a fibre was stretched to the extent that there was no overlap for most of its length, tetanic stimulation at fixed overall length caused a slow rise of tension which went in parallel with shortening of end regions where there was still overlap.

These experiments showed, in my opinion conclusively, that reliable measurements of isometric tension can be made only if the length of a uniform segment of a fibre is servo controlled so as not to be affected by shortening of other parts of the fibre with shorter sarcomeres. Gordon, Julian and I went to considerable trouble to achieve this ${ }^{5}$ and showed an almost perfect direct proportionality between isometric tension and overlap. The deviations from ideal proportionality between tension and overlap that remained in spite of servo control are explained by the residual non-uniformity of sarcomere length within the controlled segment. When at last Pollack did use servo control of a segment, ${ }^{7}$ he obtained length-tension diagrams similar to those that Gordon, Julian and I had published a quarter of a century earlier; many other investigators had also done so in the interval. In summary, the proportionality of tetanic tension to overlap is well established experimentally, and I regard this, together with shortening speed being nearly independent of sarcomere length, ${ }^{6}$ as sufficient evidence to establish the idea of "independent force generators".

A paradoxical aspect of Pollack's article is that if one were to accept the evidence from the length-tension measurements that he quotes against what he calls the "cross bridge theory", it would demolish not only the swinging cross bridge theory but also both of the theories that he puts up as competitors. Of these, Harrington's theory ${ }^{8}$ of thermal coiling in the $\mathrm{S} 2$ segment of myosin falls clearly into the class of "independent force generators" and therefore requires tetanic tension to be proportional to overlap. As regards Pollack's theory of cyclic local shortening in the thin filament, he docs not give cnough detail to make clear whether it is an "independent force generator theory" or not, but it does explicitly require overlap of thick and thin filaments. All the experiments purporting to show tetanic tension in excess of that expected from overlap also show large tension at fibre lengths where there would be no overlap if sarcomere length were uniform throughout: if one were to accept these experiments as evidence against "independent force generators", one would have to accept them also as evidence against overlap being necessary for force generation, and therefore as excluding Pollack's theory based on changes in actin - and indeed as excluding almost every theory that has been proposed since the advent of sliding filaments.

In his penultimate paragraph, Pollack raises the possibility that more than one mechanism is at work. He does not, however, mention in this context one strong reason for such an idea, namely the "repriming" phenomenon described by Lombardi and colleagues': the level to which tension recovers after two quick releases is greater if the time interval between them is, say, $10 \mathrm{~ms}$ than if it is $1 \mathrm{~ms}$, implying the operation of two processes with very different time courses. If the fast process is swinging of the cross bridges, there are several possibilities for the slow one ${ }^{10}$. shift of the myosin head to a different actin monomer, action of the second head of myosin, shortening of the S2 segment of myosin, and no doubt others. If there is a fallacy in current thinking about muscle contraction, it may be that the idea of swinging cross bridges is not actually wrong, but only incomplete. But in any case it requires stronger arguments than those put forward by Pollack to throw doubt on the more general proposition of independent force generators. There are plenty of acute questions about the mechanism of contraction but this is not one of them. 


\section{A F HUXLEY \\ Department of Physiology, University of Cambridge, Downing St, Cambridge.}

1 Huxley AF, Niedergerke R. Interference microscopy of living muscle fibres. Nature 1954;173:971-3.

2 Ramsey RW, Street SF. The isometric length-tension diagram of isolated skeletal muscle fibers of the frog. $J$ Cell Comp Physiol 1940;15:11-34.

3 Huxley AF, Peachey LD. The maximum length for contraction in vertebrate striated muscle. J Physiol (Lond) 1961;156:150-65.

$4 \mathrm{Katz}$ B. The relation between force and speed in muscular contraction. J Physiol (Lond) 1939;96:45-64

5 Gordon AM, Huxley AF, Julian FJ. Tension development in highly stretched vertebrate muscle fibres. J Physiol (Lond) 1966;184 $143-69$.

6 Gordon AM, Huxley AF, Julian FJ. The variation in isometric tension with sarcomere length in vertebrate muscle fibres. I Physiol (Lond) 1966:184:170-92.

7 Granzier HLM, Pollack GH. The desecnding limb of the forcesarcomere length relation of the frog revisited. $J$ Physiol (Lond) 1990;421:595-615.

8 Harrington WF. A mechanochenical mechanism for muscle contraction. Proc Natl Acad Sci USA 1971;68:685-9.

9 Lombardi V, Piazzesi G, Linari M. Rapid regeneration of the actin-myosin power stroke in contracting muscle. Nature 1992; 355:638-41.

10 Huxley AF. A fine time for contractual alterations. Nature 1992;357:110.

\section{From Dr T Iwazumi}

\section{It's time to go back to basics}

$\mathrm{B}$ iological science is traditionally discovery driven. In contrast, physical science and technology are theory driven. The reasons may be multitudinous. People trained in biological science are much less exposed to theoretical and abstract subjects than those trained in physical science and are forced to memorise all the details instead. Biological systems are extraordinarily complex, and thus more often than not defy the attempts of physical scientists to crack the secret of their operation. Most of the time, theories are too simplistic and inadequate and the outcomes are so far from reality that they are often the laughing stock of biological scientists, who can quickly demolish the theories by pointing out experimental facts. No wonder there are so few physical theorists who dare to venture into biological science. However, that does not mean biological science does not need physical theories. On the contrary, we are accumulating year after year millions of pieces of information and misinformation, and are desperately in need of integrating frameworks to sort out which pieces are true or false. We have become very much accustomed to seeing trees and leaves and forgetting the forest. What is needed more than ever are physical theories with clear insights that will allow mathematical descriptions of the biological phenomena. We need quantitative theories; any ideas that are not amenable to mathematical or physical descriptions are not useful at all because they are not testable. It is imperative to remember that theories are made to be destroyed, not to be upheld at all cost.

Muscle is unique among biological systems. Its behaviour is clearly definable with phyical quantities. Another advantage is its highly organised structure that can be described by mathematical terms. I believe that the first penetration of physical science into biological systems will take place in muscle. We need to realise that many fundamental issues are neglected in muscle research. In what follows I would like to point out that many of the familiar ideas in muscle research are incompatible with physics.

\section{Structural stability theory}

A human can stand on his two feet. From this observation, can we conclude that anything with two feet can stand by itself? Of course not. A dead man cannot stand on his two feet. A live man can because he is endowed with sensors and muscles to close feedback loops which stabilise him in an erect position. If any one of the feedback loops is damaged. he will fall even if he does not wish to. Is this not absurdly simple and obvious? Let us consider the structural stability of the sarcomere. Any structure, including sarcomere, subject to external and internal forces must obey a physical law which states that the structure responds in such a way as to minimise the structural potential energy (or maximise the entropy) associated with the forces. In other words, if one observes a structure under stress maintaining a certain shape over a time period longer than the transient time (that is, the structure is not in a process of an explosion), the structure is in quasi-equilibrium. Figure 1 shows a familiar side view of a sarcomere. The factual observation is that the thick filaments are situated in the middle of the sarcomere during relaxation as well as during steady isometric contraction. We know nothing about the nature of the forces, but we are sure that forces are acting between the thick and thin filaments. Now let us assume that the longitudinal force (along the long axis of the sarcomere) is proportional to the overlap length between the thick and thin filaments. Then any slight shift of the thick filaments to the right from the centre (due to thermal agitations) will result in a greater force in the right
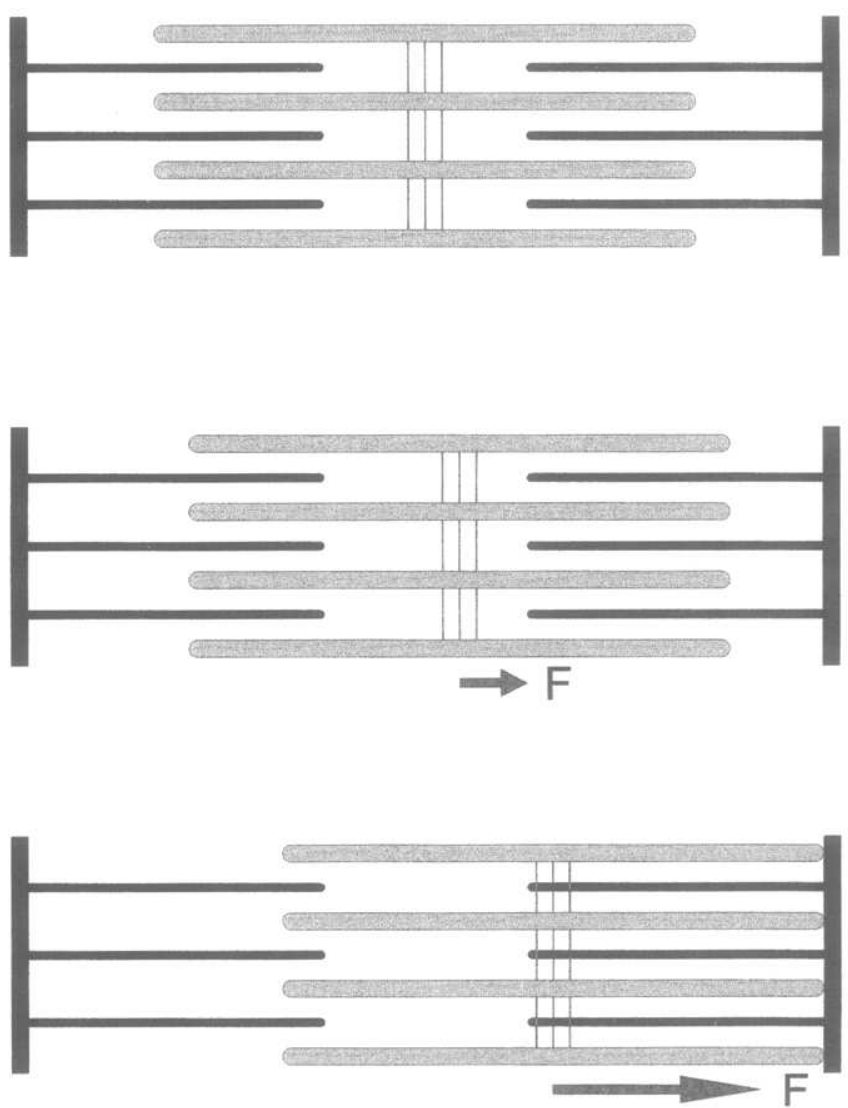

Figure 1 Longitudinal instability of all mechanisms invoking proportionality of the axial force with the overlap length between thick and thin filaments. One cannot circumvent this global instability by hoping that local stability will play miracles. 
half and a lesser force in the left. The more shifts to the right, the more forces to the right; therefore, the thick filaments will hit the right hand $\mathrm{Z}$ disc. However, this is not what we actually observe. To remedy the problem, let us further assume that position sensors and activation controllers exist on each side so that when the thick filaments begin to shift to the right, the force on the right is reduced and the force on the left increased. This is a stabilisation control system similar to the one that allows a human to stand on his two feet. Cross bridge theorists ${ }^{12}$ believe that each cross bridge is endowed with this property. That is, cross bridge forces diminish when the thin filament moves forward and are cnhanced when it moves backward. However, this argument works only within a small range. Any sensor or actuator has a limited working range. For example, in the case of the human standing on his two feet, he can only maintain balance within a limited angle. For a sarcomere containing thousands of cross bridges cycling randomly, such precarious balances cannot be maintained in wide ranges. An important point here is that the stabilisation effect must persist in the entire range of overlap. This is called global stability. In contrast, the stability afforded by each cross bridge is called local stability. An intuitive example representing a system with global instability and local stability is a ball placed on a mountain having many small dents on the slope. The ball may dwell in a dent temporarily but falls off the mountain anyway because the mountain is shaken all the time (by thermal agitations). It would be interesting if someone attempts a computer simulation of a sarcomere with a large number of cross bridges to see how long the thick filaments stay put in the middle. The possibility that the thick filaments are supported by elastic structures to withstand any unbalanced forces is untenable because such structures result in a very stiff sarcomere. A formal mathematical proof of the longitudinal stability condition (in a global sense) can be given by applying Liapunov's second method. ' Note that not all mechanisms which result in proportionality of the force with the overlap length can satisfy the longitudinal stability condition. The nature of the force is irrelevant. It does not matter whether the force comes from cross bridge cycling or electrostatic interaction or melting of protein structure. The longitudinal stability condition alone eliminates a vast majority of proposed mechanisms of muscle contraction because they were specifically designed to have that property due to a misinterpretation of experimental data.

Another structural stability condition must be satisfied in the sarcomere for the sliding filament mechanism to work properly. It is lateral stability of the thin filament. Figure 2 shows the cross sections of the unit cell formed by four thick filaments as observed in three different types of muscle. In the figure, the large filled circles are thick filaments and the simall ones thin filaments. Depending upon the number of thin filaments in a unit cell, the locations of thin filaments are different. The functional significance of such arrangements is obvious. Thin filaments must slide freely in the small space surrounded by thick filaments. If the thin filaments are allowed to cross the unit cell boundary, the ensuing chaos will make the sarcomeres inoperable. A crucial question is how are such positional stabilities achieved depending upon the number of thin filaments in a unit cell. A simple idea of mutual repulsion force between thick and thin filaments will not work because the location in fig $2 \mathrm{~B}$ is unstable under repulsive forces.

The lateral stability condition has never been addressed by all proposed muscle theories except one. Everyone has taken thin filament lateral positioning for granted without asking why. Cross bridge theorists presumed that cross bridges bind the thin filament in a fraction of their cycling time, and that the binding sites along the thin filament are widely spaced compared with the reaching distance of the cross bridge. If so, the thin filament has virtually no lateral support at all. How do we expect thin filaments to know where they belong? One can stretch and release sarcomeres many times while they are in relaxed (inactive) state and never see the thin filaments stray away from their respective unit cells. Inactive sarcomeres are remarkably stable structures. There exists only one theory ${ }^{3}$ that elegantly solves this problem.

\section{Stiffness theory}

It has long been known that muscle stiffens when it goes into contraction. Except for a few cases, all muscle stiffness measurements have been made in the longitudinal (axial) direction. The stiffness is derived from the force responses to either small vibrations or step length changes applied at one end of muscle. Consistent findings are that muscle longitudinal stiffness increases roughly in proportion to the contractile force produced. These findings have been interpreted to be a result of cross bridge linking to the thin filament. The greater the force, the greater the number of linkings, and therefore, the greater the stiffness. However, in 1982 Tamura et $\mathrm{al}^{4}$ reported a strange phenomenon. They found that longitudinal stiffness increased with contractile

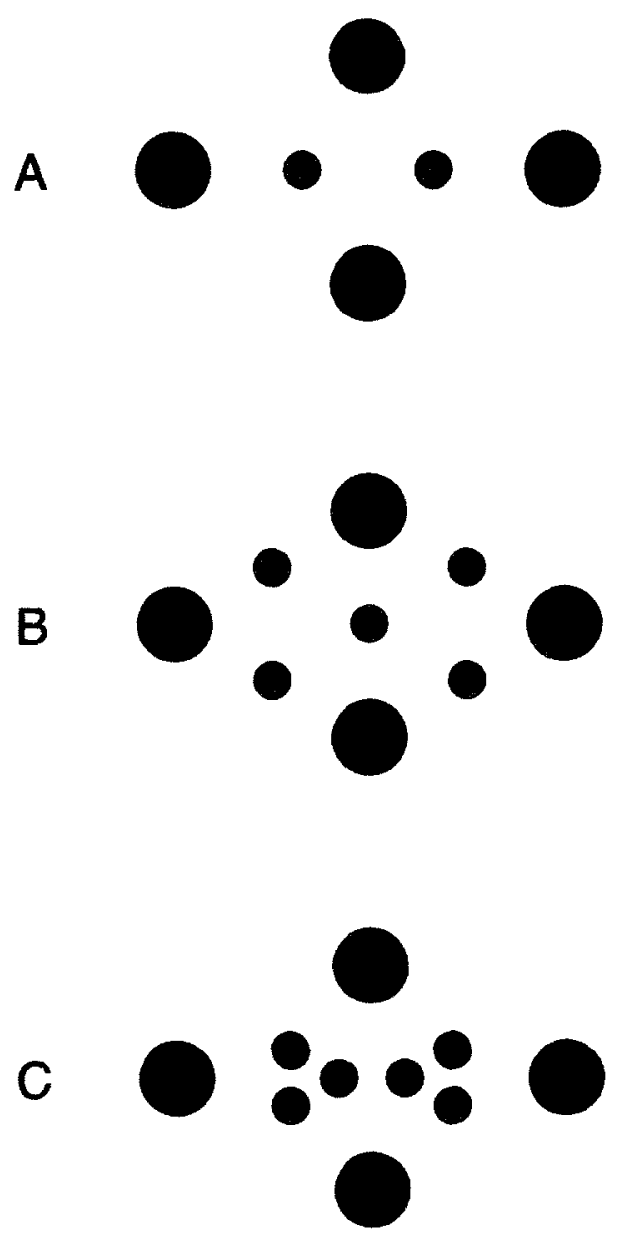

Figure 2 Cross sectional views of three different types of muscle unit cells. Panel $A$ is of skeletal muscle, $B$ is of insect fight muscle, and $C$ is of insect leg muscle. The pattern $B$ is most stable, which excludes the possibility of electrostatic repulsion as a primary mechanism for these patterns. 
force, as everyone else did, but that the lateral stiffness measured across the muscle decreased instead. If the longitudinal stiffness increase is due to cross bridge linking, the lateral stiffness must increase as well. Nobody could offer an explanation for their findings and the paper was quickly forgotten. One possible solution to this dilemma might be found. Suppose that cross bridges are linked to the adjacent thick filament (or the cross bridges from it) while in relaxation, and also linked while cross bridges are detached from the thin filament during cycling. In other words, if the cross bridge jumps between thick filament and thin filament during contraction, Tamura's experimental fact might be explained. The only problem is that nobody has ever found that cross bridges bind to thick filaments or to themselves during relaxation. This is another impossible situation for the cross bridge theory to explain, alongside the structural stability problem discussed in the previous section. Cross bridge theory should have been dead since 1982 when people could not explain the decrease in lateral stiffness during the increase in longitudinal stiffness.

Let us consider then if other ideas of contractile mechanisms might explain Tamura's experiment. If the forcc is produced by melting of a part of myosin, ${ }^{5}$ the longitudinal stiffness ought to show very complex behaviour; it would have high stiffness at low force (partial melting) and low stiffness at high force (full melting), yet it would stiffen when stretched. The behaviour would be far from a simple proportionality relationship found in muscle experiments. We must see experimental evidence of protein melting resulting in a simple proportionality relationship between force and stiffness before accepting the melting as a candidate of the contractile mechanism. The same problem occurs for the latcral stiffness as long as the basic mode of operation of the melting protein is cross linking between the thick and thin filaments. Increased cross linking never results in decreased stiffness; it is physically impossible.

What is the physical meaning of increasing stiffness in one direction and decreasing stiffness in another? Any substance, be it homogeneous (isotropic) or composite (anisotropic), has a positive stiffness in a given direction. Muscle is highly anisotropic due to its highly orientated internal protein structure. Water is isotropic and its contribution to the muscle stiffness is small (water stiffness, as well as protein stiffness, is frequently dependent). Therefore, the stiffness we observe comes mostly from the protein structure. A physical meaning of positive stiffness can be understood by considering a coiled spring. A spring stores potential energy when it is deformed from unrestrained length by an external force. As shown in fig 3, a derivative (or gradient) of the potential energy with respect to the distance is related to the force (a vector). Again, a derivative of the force with respect to the distance is related to the stiffness (a tensor). With due care paid in choosing the direction of the force (to take care of a minus sign), we see that the stiffness is a second derivative of the potential energy curve. In other words, a positive stiffness means that the potential energy curve has a concave upward shape. When muscle is in relaxed or resting state, it has certain stiffness values along longitudinal and lateral directions. As muscle goes into contraction, something changes inside and we observe longitudinal stiffness increase and lateral stiffness decrease. That "something" produces a positive stiffness in the longitudinal direction and a negative stiffness in lateral direction(s). In terms of energy, positive stiffness means that the object is absorbing energy from outside by deformation, and is releasing energy if negative. There are only a few examples of negative stiffness in nature; one is of gravity field and another is of electrostatic and magnetic field, in particular dipole-dipole interaction. Dipole-dipole interaction is unique because it can be either energy absorbing or energy releasing depending upon the mutual orientation of dipoles. Figure 4 shows four simple cases of dipole-dipole interaction; negative potential energy releases energy and positive potential energy absorbs it from outside. When the mutual orientation of the dipoles is intermediate, we get all possibilities of complex potential energy curves. An example is shown in fig 5 . We see that the potential energy curve is concave downward resulting in negative stiffness which is not constant. An important point is that the magnitude of the
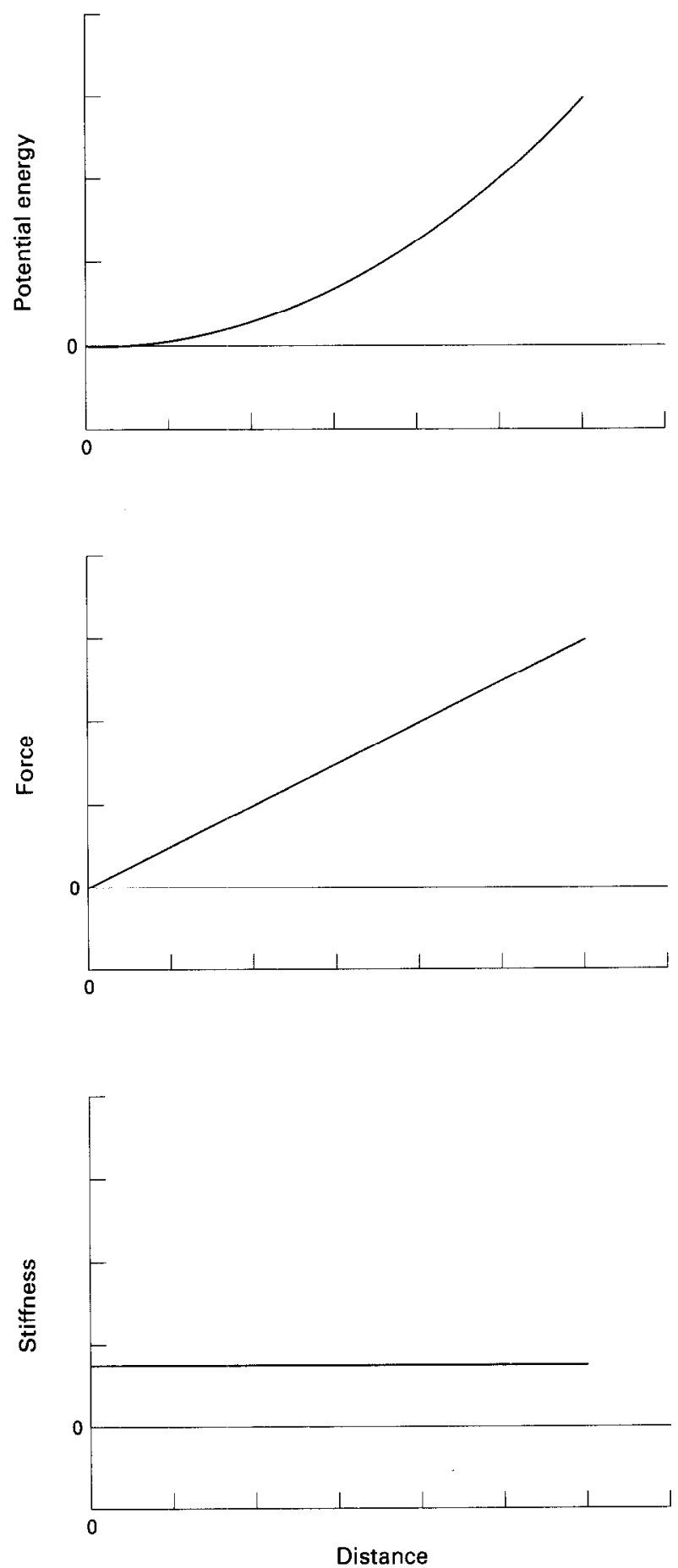

Figure 3 Relationships between potential energy, force, and stiffness of a mechanical spring. The force is a derivative of the potential energy, and the stiffness is a derivative of the force. 

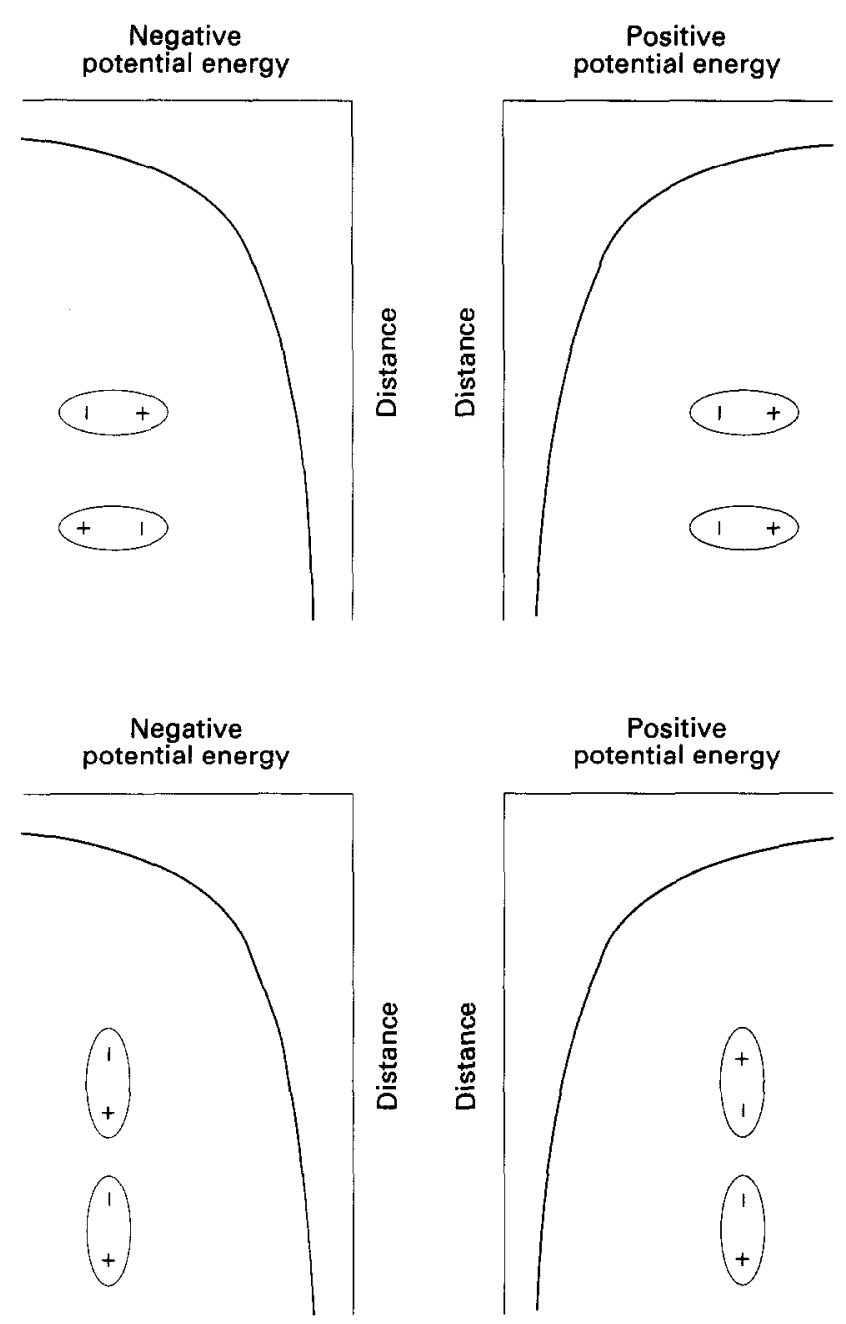

Figure 4 Potential energies of dipole-dipole interactions. Organised dipole structures can produce complex potential energy curves with interesting properties.

negative stiffness changes with the magnitude of the potential energy which is directly related to the dipole energy; the greater the dipole energy, the greater the negative stiffness. Since the contractile force is directly related to the dipole energy, the magnitude of the negative stiffness is directly related to the force, thus resulting in decreased lateral stiffness. The potential energy curve in fig 5 was taken from my work 25 years ago ${ }^{3}$ which describes in detail how dipoles are arranged along the thick filaments.

The longitudinal stiffness in muscle has been found to increase with contractile force in most experiments, but this is not always the case. Under some special conditions, that are unphysiological in skeletal muscle but proper in insect muscle, longitudinal stiffness can also decrease. The theory ${ }^{6}$ of longitudinal stiffness is very complex and inappropriate to discuss here.

\section{Conclusions}

One of the reasons why muscle research is so difficult may be attributable to the problem of quality control of muscle preparations. Preservation of the entire sarcomere structure throughout the experiment is a paramount requirement because the mechanical behaviour depends strongly on its structural integrity. Unfortunately, the vast majority of published data do not even discuss to what extent an effort was made to preserve sarcomere patterns during activation.
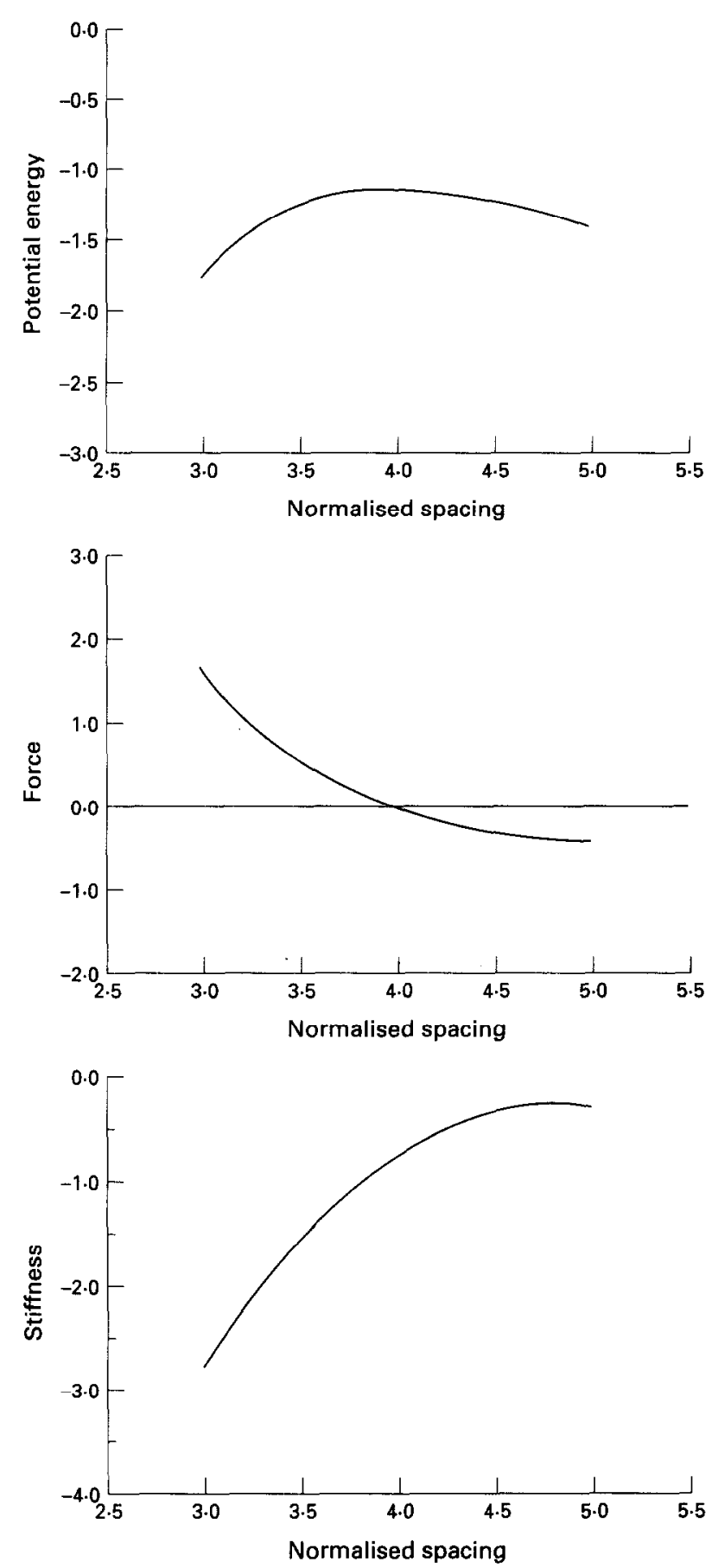

Figure 5 Lateral potential energy, force, and stiffness of a helical dipole array. Note that the force can assume bipolar values; for the normalised spacing less than about 4, the array tends to shrink, but above 4 it expands. However, the stiffness is always negative and is proportional to the dipole energy (that is, negative stiffness increases with the strength of the dipoles).

Preservation of sarcomere patterns is particularly important for demembranated muscle experiments where sarcomere patterns are usually lost at the first activation. Most sarcomeres cannot maintain structural integrity if exposed to calcium concentration greater than a few micromoles. If one in three of the sarcomeres in the preparation is damaged, the sarcomeres as a whole will still look reasonable, but the mechanical properties will be greatly altered. Because of a historical belief arising from cross bridge theory that sarcomeres must be supramaximaly activated to make sure that all cross bridges are in action, most sarcomeres were destroyed at the very first activation. The loss of sarcomere patterns 
during contraction is proof of their destruction. All the data taken from destroyed prcparation are, of course, invalid. To minimise the effect of damaged sarcomeres, the use of preparations consisting of only a few sarcomeres would be advantageous, since we can then observe the behaviour of every sarcomere during the experiments. Nevertheless, the process of reducing a muscle fibre to such a small myofibril preparation itself causes damage to the sarcomeres. At present, the only solution is to repeat vast numbers of trials until a high quality preparation is obtained.

Because of the general popularity of applying molecular biology techniques in recent years, people tend to forget the crucial importance of organised structure. One must remember that the results obtained in elementary molecules cannot be directly extended to the organised structure unless the working of the structure is well understood. Understanding the function of a transistor has nothing to do with understanding the function of a computer. We must devise experiments to preserve sarcomere structure and function as intact as possible. Sarcomeres should be regularly spaced and squarely registered and should show no distortion during contraction by avoiding excessive activations. For the future of muscle research, we have two choices: either disregard of physics (continuation of fantasy) or respect for physics (a revamp of the whole muscle research). Negligence of physical principles in muscle research is appalling, particularly in the lack of understanding vector and tensor. No matter how much we investigate the biochemistry of muscle, there is no possibility of finding vectors and tensors from biochemistry. What is critically important in muscle research is to find a bridge between chemistry (scalar) and physics (vector). To do so requires that we must have physically possible ideas to start with. We have wasted vast amounts of resources and effort in pursuit of physically impossible ideas for a quarter of a century. Enough is enough.

T IWAZUMI

Albert Einstein College of Medicine, Division of Cardiology, Bronx, New York, USA.

1 Huxley AF. Muscle structure and theories of contraction. Prog Biophys Chem 1957;7:255-318.

2 Huxley HE. The mechanism of muscular contraction. Science 1969;164:1356-66.

3 Iwazumi T. A new field theory of muscle contraction. Pennsylvania: University of Pennsylvania, 1970. $\mathrm{PhD}$ Dissertation.

4 Tamura Y, Hatta I, Matsuda T, Sugi H, Tsuchiya T. Changes in muscle stiffness during contraction recorded using ultrasonic waves. Nature 1982;299:631-3.

5 Harrington WF A mechanochemical mechanism for muscle contraction. Proc Natl Acad Sci USA 1971;68:685-9.

6 Iwazumi T. Molecular mechanism of muscle contraction. Physiol Chem Phys Med NMR 1989;21:187-219.

\section{From Professor S V Perry}

\section{Don't burn all the bridges}

$\mathrm{T}$ The precise description in molecular terms of the contractile mechanism in muscle is not yet available. The dogma in this field has been that the relative movement between the A and I filaments is the result of a cross bridge cycle during which the hydrolysis of ATP provides the energy for force production. Gerald Pollack has never been happy with this view and does some service by bringing together all the evidence which he considers does not support the current "establishment" position. This evidence must be appraised and if its validity is confirmed, reconciled with the dogma. Nevertheless he does a disservice to those not closely associated with the field if he gives the impression there is much support for the views he presents.

There is little doubt that the contractile activity in muscle is the consequence of the interaction between the proteins myosin and actin and that the motor protein is myosin. This interaction is much more subtle than that envisaged by the original simple swinging cross bridge theory. It is probable that the two proteins are in contact at all times. The current view is that there is a weak interaction between these proteins in the relaxed state which changes to a strong force developing form on stimulation. This results from the conformational changes occurring in the myosin molecule associated with the hydrolysis of ATP. These molecular changes cause an adjustment of the position of the myosin head between adjacent actin monomers of the I filament. To accommodate this adjustment, relative movement between the filaments takes place.

The field has recently been illuminated by the elucidation of the structures of actin ${ }^{1}$ and the myosin head, ${ }^{2}$ but we are still only able to speculate as to the conformational changes in these molecules that give rise to relative movement. The model based on the myosin structure proposed by Rayment et $\mathrm{al}^{3}$ suggests that the power stroke involves a $6 \mathrm{~nm}$ movement of the long tail of the head of the myosin molecule relative to the body of the head. As Pollack points out, all attempts to determine conformational changes in the myosin head accompanying contraction have becn disappointing in that they do not appear to be very pronounced - certainly not enough to accommodate the changes suggested by the model. Nevertheless the information now available about potential sites of interaction between the two proteins, coupled with the availability of model motile systems for testing forms of actin and myosin obtained by site directed mutagenesis, means that answers to these questions should soon be available.

Despite the uncertainties regarding the precise details of the mechanochemical process, the evidence that the motor activity resides in the head of the myosin molecule is most impressive, almost irrefutable. The actomyosin system for the transduction of chemical energy into force development and movement is widespread in cells. Muscle, the most highly specialised contractile tissue, contains a double headed myosin molecule with a long coiled coil $\alpha$ helical tail, which Pollack wishes us to consider as site of the motor unit. This form is now called myosin II (because of its double head) to distinguish it from at least seven other types of Inyosin which have been identified. ${ }^{4}$ These are known collectively as the unconventional myosins and are involved in general cell activities such as the movement of particules, membranes, and vesicles about the cell, cytoplasmic streaming, pseudopodia extension, budding, and many other widespread cellular functions that depend on motility. Molecular genetic studies indicate that all myosins have probably evolved from some primitive precursor molecule with contractile function. Unconventional myosins are identified by consensus amino acid sequences in the head region that are essential for motor function and its regulation. They differ in the number of heads and the length of the tail region; indeed in some the tail is absent altogether. Unlike the head region there is no homology in the sequences of the tail regions of the different myosins, making it extremely unlikely that this region of the myosin has a common role 
which would be required if it was involved in the transduction process. The present view is that the tail has a role related to the "cargo" function of the motor, that is, to attach the motor to the filament, membrane, or vesicle, for example, that it moves, but not to generate force. In view of this wealth of information and of the fact that from the results of work with model motile systems with tailless fragments of the myosin molecule, it is difficult to make any case for a significant role for the myosin tail in the energy transduction process. It is possible, however, that the hinge region in the tail of myosin II could in some way modulate the contractile response of the motor.

Even Pollack would find it difficult to argue against the conviction that ATP provides the energy for force production in the muscle contractile system. As is the case with myosin II, the unconventional myosins possess ATPase activity that is located in the head of the molecule and activated by actin. In cells virtually all the ATP is present as the magnesium complex, the substrate for most enzyme systems which handle ATP. In contrast MgATP is a very poor substrate for the myosin ATPase, with a rate constant of about $0.1 \mathrm{~s}^{-1}$. When actin interacts with myosin the rate constant for ATP hydrolysis is increased by two orders of magnitude, that is, the motor is turned on. In resting muscle the motor is turned off by preventing actin from interacting with myosin. Activation of all muscle types involves relief of the inhibition of this interaction, leading to a high rate of ATP hydrolysis. Much is known about the intracellular control of contraction in muscle; indeed in some ways the regulatory mechanisms are better understood than the transduction process itself. The manner in which the calcium transients control contractile activity varies in different muscle types but in all cases the final stage in the sequences of events would appear to be modulation of the interaction of actin with the myosin head. Thus the myosin motor, in addition to its ability to convert chemical into mechanical energy, is designed with a built in switching mechanism. This unique property of myosin is essential for the contraction-relaxation cycle. Very similar properties are associated with the tubulin based motor proteins, dynein and kinesin. Those who attack the view that the motor for contraction is located in the myosin head all too often forget, or are unaware of, the compelling evidence of its location provided by the study of the regulatory processes in muscle. In their alternative models they rarely consider this problem. Contraction without control has no meaning in a living system - in muscle it leads to rigor mortis.

Although there is no evidence for significant changes in periodicity in the actin filament, small molecular state transitions in the actin monomers in functioning muscle could take place. They are most unlikely to be responsible for the shortening process for the reasons already presented. Furthermore all the evidence is that actin provides the relatively inert protein track along which the motor moves. It is a strongly conserved protein and the actins used by the different myosin motors are very similar in amino acid sequence and therefore function. On the other hand the myosin motors show a wide range of properties which are appropriate for their different functions. It is more likely that any propagated molecular state transitions in the actin filament are a consequence of the regulatory process. They could arise as the result of the interaction at defincd site(s) on actin of myosin and of troponin $I$ in the contracting and relaxed states respectively. One of the intriguing problems of striated muscle is that all of the actin monomers of the I filament in resting muscle are unable to activate the myosin motors. This is despite the fact that troponin I, the protein blocking the interaction of actin with myosin in resting muscle and thus inhibiting the ATPase, is only located on every seventh actin monomer. In some way this effect must be conveyed to the adjacent six actin monomers with which troponin I is not directly associated. One could easily visualise the conformational change occurring when troponin $I$ binds to one actin monomer being transmitted directly to neighbouring monomers in the I filament so that they too are unable to activate the myosin motor. Alternatively it can be postulated that the tropomyosin lying in the groove of the I filament blocks the interaction site for myosin on actin in resting muscle. On stimulation it is suggested that the tropomyosin moves, leaving the interaction site on actin free, the so called steric hypothesis much promulgated in the textbooks. Pollack could have spent his time more profitably examining this dogma.

The answer to the question he poses is clearly no. "Methinks he doth protest too much".

S V PERRY

Department of Physiology,

The Medical School, University of Birmingham,

Birmingham, UK.

1 Kabsch W, Mannherz HG, Succk D, Pai EF, Homles KC. Atomic structure of actin: DNAse complex. Nature 1990;347:37-44.

2 Rayment I, Rypniewski WR, Schmidt-Base K, et al. Three dimensional structure of myosin subfragment-I: a molecular motor. Science 1993;261:50-8.

3 Rayment I, Holden HM, Whittaker M, et al. Structure of the actinmyosin complex and its implications for muscle contraction. Science 1993;261:58-65.

4 Cheney RA, Mooseker MA. Unconventional myosins. Curr Opin Cell Biol 1992;4:27-35.

\section{From Professor Marcus Schaub and Dr Daniel Koch}

\section{Actin motor versus myosin motor in muscle contraction}

I n Pollack's article on the muscle contraction mechanism he poses the question: "Are alternative engines gathering Lsteam?". He then goes on to propose an actin based motor as an alternative engine in opposition to the myosin motor. The contractile mechanism touches on a most fundamental biological question: how is one form of energy transformed into another? In the case of motility, how is chemical energy transformed into directional mechanical energy? In the case of photosynthesis, for example, it is by the conversion of radiation energy into chemical energy. On the molecular level energy transduction follows through a succession of protein conformational changes coupled to enzyme kinetics. ${ }^{2}$

The swinging myosin cross bridge model is enjoying a long standing reputation and has, indeed, made its way into the textbooks. Suffice it to mention the two epoch making publications by A F Huxley and $\mathrm{H}$ E Huxley and their respective coworkers, ${ }^{3}$ which appeared in the same issue of Nature in the mid-fifties and which introduced the sliding filament theory of contraction. This theory was arrived at by careful and lucid interpretation of experimental results obtained by refined and new methodological techniques. It caused a shift of paradigm since the concept of two intracellular filamentous protein structures which bring about muscle shortening by sliding past each other was contrary to the commonly held view at that time. It was thought that macroscopic movement results from melting of rigid and extended protein structures such as helix coil transitions. 
Such conformational changes entail a transition from highly ordered to much less ordered randomised structures as observed by increasing temperature, or by changes in $\mathrm{pH}$ or in ionic environment. The energy liberated by these processes was thought to drive movement. Restoration of such processes, coupled with changes in $\mathrm{pH}$ or ion composition over larger intracellular areas, would however be costly and time consuming. It is hard to see how movement could be driven when almost as much, or probably more, energy is required to restore the original state, ready for contraction again. A corollary to the sliding filament theory was the concept of independent force generators which must be distributed along the interdigitating filaments. ${ }^{5}$ These force generators have come to be known as myosin subfragment-1 or myosin head in which reside the hiological properties such as ATPase, actin binding, and motor activities. The myosin heads form the cross bridges which by some sort of movement displace the actin filament toward the sarcomere centre. It is still beyond the possibility of resolution to discern whether the two myosin heads of one molecule are combined in one active cross bridge or whether they operate independently of one another.

The further development and refinement of the myosin cross bridge theory and its implications for function in muscle and non-muscle cells makes for a breathtaking scientific story when compressed into a short comment. In reality, it represents a period of worldwide research over four decades with the individual contributions scattered over almost 20000 publications, that is, between one and two reports a day. Being aware that only a marginal number of scientific reports in any field are making a significant impact, for example, less than $10 \%$ of them get cited more than once, it scems, neverthcless, that most of them are valuable in setting up the body of evidence that allows the prevailing paradigm to be built upon.

Research in muscle contraction has always involved a multidisciplinary approach, mainly in the fields of biochemistry, physiology, and structural biology. The results from the different disciplines were overviewed for the first time at the memorable Cold Spring Harbor Symposium of 1972 on "The Mechanism of Muscle Contraction". The cross bridge theory was supported in all its implications by the then available methodology. The swinging cross bridge paradigm has such a compelling power that it makes it difficult to look for alternatives. Indeed, one tends to find the results one is hoping for. Pollack must be credited for his open minded approach. In view of the broad consensus on the myosin motor function the proposition of an actin based motor seems rather extravagant. It rests on a small basis of experimental evidence $^{l}$ compared to the classic cross bridge model. Nevertheless, it requires serious consideration. In addition, following up the idea and consequences of the actin motor model is the fun of it. Independent of its viability, the exercise may sharpen critical evaluation of the classic contraction mechanism as well as further unconventional thinking.

\section{Does the actin based motor live up to expectations?}

To start with, role is not merely passive. Chemical modifications and specific point mutations in actin are known to affect cross bridge kinetics and in vivo muscle function, for example flightless variants of Drosophila melanogaster or the uncoordinated movement of Caenorhabditis elegans. ${ }^{7}$

Filamentous actin can exist in two different states, that is, in helical and ribbon stranded structures, and it has been suggested that the wave-like transition between them is involved in force generation during muscle contraction. ${ }^{8}$ Following this line, Pollack proposes that within the actin filament the monomers may exist in a "long" or in a "short" state. ${ }^{1}$ Transition of the monomers from "long" to "short" would result in a shortening of the entire actin filament to some small degree. During muscle activity the myosin heads are supposed to interact with the actin filament. The long actin monomers start shortening rapidly, beginning from the barbed end at the $\mathrm{Z}$ line of the sarcomere. This transition would run up to the point where overlapping with the myosin filaments begins. The oncoming front of actin monomer shortening is slowed down by the attached cross bridges. Since "short" actin has a low affinity for the myosin head, the cross bridges detach successively as the actin transition proceeds. The detachment rate of the cross bridges may be governed by the rate at which ATP is hydrolysed. The transition front keeps progressing toward the pointed end of the actin filament, albeit at a slower pace. This process is supposed to advance the actin filament as a whole toward the centre of the sarcomere. As a consequence the sarcomere should be shortened. Subsequent recovery of the actin filament 10 its initial state may be induced by a reverse lengthening of the monomers, again starting from its barbed end at the $Z$ line. The lengthening transition is following behind the shortening transition and allows the cross bridges to reattach as the myosin heads have a high affinity to the "long" state of actin (see fig 2 in '). So waves of transition may follow one another travelling fast in the non-overlap region and slowly in the overlap region, until contractile activity subsides. So far Pollack's premises. As elegant as this model appears at first, it may encounter problems when put to the test.

The transition from the "long" to the "short" state could entail a reorientation of the monomers, with their long axis approximately parallel to the long axis of the helical $F$ actin into the ribbon structure. In the ribbon structure actin monomers are orientated with their axis diagonally, in an alternating fashion, with respect to the filament axis. ${ }^{8}$ This could result in an overall shortening of the filament by a few percent. Since the actin filaments are anchored on the $\mathrm{Z}$ line, such a transition is postulated to start from there. The signal which may initiate the transition from "long" to "short" is not known. Nor is it known how the reverse transition, back to "long", gets started at the $\mathrm{Z}$ line which must follow some time later. How could such a contractile mechanism work?

(1) First of all, Pollack's proposal relies on intermittent contacts between the actin filament and myosin cross bridges. ${ }^{1}$ Without that the actin filaments would oscillate between "long" and "short" states, neither moving the $\mathrm{Z}$ line toward the sarcomere centre nor producing force. At rest the muscle is slack with most of the cross bridges detached, so that it can easily be stretched passively. Upon activation the cross bridges must make contact with actin first in order to provide the grip. Also in this case the regulatory switch will most likely be mediated by binding of calcium to troponin $\mathrm{C}$, as in the swinging cross bridge model. Whether this switch induces the actin filament transitions at the same time seems questionable. These latter transitions must originate from the $\mathrm{Z}$ line in order to ensure the correct directionality. Hence a second signal must be postulated at the level of the $\mathrm{Z}$ line. The question then arises, what is the nature of this second signal and how is it coupled with the first one? Such a second signal needs to operate on the millisecond time scale akin to the calcium switch of the troponin-tropomyosin system. A signal involving either covalent protein modification like phosphorylation, or enzymatic production of a 
second messenger, is out of question. Furthermore, such a fast second signal would be required at a narrowly confined site at the $\mathrm{Z}$ line. No other possibility may be left but to make use of the same calcium signal which is able to trigger contraction at the required speed. In this latter case how can the two processes (attachment of cross bridges and induction of actin transitions) be tuned temporally in the right order? Alternatively, the actin filaments may oscillate between their two states continuously, whether the cross bridges are off or on, that is, at rest as well as during contraction. This latter alternative looks like a less efficient arrangement than one would expect from a highly specialised motor apparatus. It would consume constantly a considerable amount of energy, not much less than that required for unloaded shortening; consider that in skeletal muscle the energy consumption jumps up by a factor of three orders of magnitude in going from rest to active shortening.

(2) Initially, the shortening transition of the actin monomers starting from the $\mathrm{Z}$ line travels fast to the nonoverlap region. This results in shortening of the entire actin filament, or at least a significant portion of it. Since the actin filament is still being held in the overlap zone by the cross bridges, the $\mathrm{Z}$ line is pulled toward the sarcomere centre by as much as the actin filament was shortened in the nonoverlap. As the shortening wave has arrived at the overlap region, it gets slowed down by the attached myosin cross bridges. Because it is now proceeding slower the cross bridges are detaching progressively. The detachment rate, and therefore also the wave propagation, are governed by some step in the hydrolysis cycle of the myosin heads. The detached cross bridges do not readily reattach to the "short" actin monomers because of low affinity for them. Some moment later the reverse transition (from "short" to "long") may start from the $Z$ line. As the lengthening wave is travelling through the non-overlap region the shortened actin monomers get extended again. Since the shortening wave in front is making slow progress in the overlap region, the pursuing lengthening wave reduces the gap between them. $\Lambda$ s a consequence, the parcel of shortened actin monomers travelling between the two approaching waves gets smaller and smaller. As long as the lengthening wave has not arrived at the overlap region, none of the detached cross bridges is able to reattach. Only when the lengthening wave goes past the beginning of overlap does the affinity to the newly lengthened actin monomers increase and cross bridges can reattach. This marks the moment when the actin filament becomes fixed at the non-overlap to overlap boundary. This fixation may serve as resistance against both directions. The tip of the actin filament now only advances toward the sarcomere centre by exactly the distance which can be recovered by the lengthening transition of those "short" monomers which managed to pass beyond the beginning of overlap. This results in a longer region of overlap and hence a shortening of the half sarcomere. This process may be repeated as many times as necessary to produce a macroscopic muscle shortening.

On the other hand, all "short" actin monomers in the nonoverlap region, which never entered into the overlap zone, revert to the "long" state causing the $Z$ line to slide back. The amount of sliding back of the $\mathrm{Z}$ line could be reduced by letting the lengthening transition follow the shortening wave after a short interval. But the shortening would also become almost negligible in that case. Or else, the more the lengthening transition is delayed behind the shortening wave the greater is the danger that too many cross bridges detach over a long stretch of the overlap region, leading to instability of the sarcomere. The parcels of shortened actin monomers which travel along the filament may vary in size. Nevertheless, all actin monomers in the non-overlap region must undergo such an energy consuming transition even if they don't reach the overlap zone. It needs emphasising that the non-overlap is usually comparable to the overlap region in length. Therefore a significant amount of actin monomers is using up energy in vain. Overall, it looks as if this would be a very inefficient contractile mechanism - one step forward and two steps back. In contrast, in the classic model every active cross bridge in the overlap zone contributes to the force production.

(3) In the classic model an increase in isometric force production is achieved by recruiting more and more active cross bridges by reducing their detachment rate relative to the rate of attachment. This increases the duty cycle, defined as the fraction of the total cycle the cross bridge spends attached to actin in a motion generating state. At high loads individual cross bridges may slip back, but they are able to reattach quickly and produce force again. Near the point of breakdown more and more cross bridges may slip with intermittent reattachments and eventually the muscle will yield slowly. In rigor, the cross bridges are firmly attached to the actin filament without producing active tension. When the critical point is reached and cross bridges begin to slip off they cannot redevelop active force; so the continuous structure between actin and myosin filaments breaks down like a house of cards. If the force developing capacity resides exclusively in the actin filament structure, the behaviour at the critical point may more closely resemble the situation in rigor. The critical point during isometric contraction will he reached when the tendency of the actin monomers to transform from "long" to "short" in the non-overlap zone is just offset by the resistance the muscle encounters. Therefore a shortening wave which could enter the overlap region does not build up at all. Only the actin monomers in the nonoverlap zone are able to contribute to isometric force, since those in the overlap zone are kept in the "long" state by the attached cross bridges. Slipping of the first few cross bridges will weaken the sustaining structure of the overlap zone. Some of the detached cross bridges may reattach. But since they are not ahle to produce active force a breakdown in the overlap region follows.

\section{The power stroke of the myosin cross bridge revisited}

Recent years have seen a revolutionary development of novel methodologies in structural biology, in vitro motility assays, and mutagenesis, leading to a new understanding of the molecular mechanism of myosin motor function. We would like to draw attention to some of the highlights and pose the question whether the new insights require a shift in paradigm of the cross bridge theory again.

The integration of the three approaches has provided considerable support to the mechanochemical coupling of the actin activated ATPase activity and the power stroke of the myosin cross bridge. ${ }^{9}$ Myosin exists in two states, one with high and the other with low affinity for actin, which are governed by the bound nucleotide, ADP or ATP, respectively. ${ }^{2}$ While myosin is bound with high affinity to actin, again at least two more states can be discerned, one before and the other after the power stroke (figure). Because myosin is anchored in the myosin filament the power stroke gives rise to about a $10 \mathrm{~nm}$ displacement of the actin relative to the myosin filament. Subsequent dissociation of ADP allows rapid binding of ATP followed by detachment of the cross bridge, and the contraction cycle may repeat. 


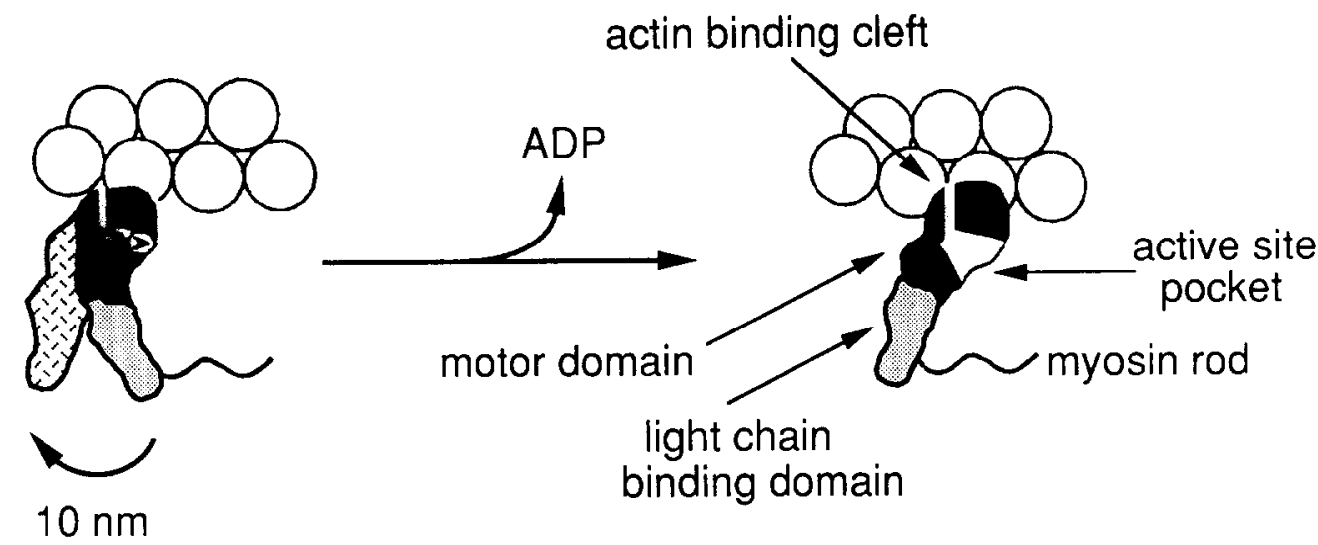

Schematic representation of the myosin power stroke. Phosphate release is thought to induce a movement in the motor domain (left side). This movement is amplified by the lever arm (light chain binding domain; arrow indicates displacement of around 10 nm) and accompanied by $A D P$ release. For convenience only one myosin head is shown attached to $F$-actin (adapted from Rayment et al, reference 12 ).

A milestone was the elaboration of the three dimensional crystal structure of the myosin head two years ago. ${ }^{10}$ Together with the known three dimensional structure of actin $^{11}$ it was possible to recognise the actomyosin interaction site on the submolecular level. ${ }^{12}$ Two particular protein surface loops have been described, one (amino acid residues 627-646, chicken sequence) involved in the actin binding region and the other (residues 204-216) associated with the active site pocket. These two loops are quite variable among different myosin isoforms. Chimaeric myosins have been constructed by site directed mutagenesis. ${ }^{9}$ Loops (627-646) from different mammalian myosins were incorporated into myosin from Dictyostelium discoideum. The actin activated ATPase activity was similar to the native homologous myosins from which the loop sequences were derived. Also the velocity of actin filament translocation in the in vitro motility assay reflects similar differences to a lesser extent. The significance of the second loop (204-216) at the active site is not yet clear.

Actin displacement requires a small conformational change in the globular myosin motor domain which is then amplified by the stalk of the light chain binding domain (figure). The stalk of the myosin head consists of an extended $\alpha$ helix of the heavy chain which is stabilised by the two light chains (essential and regulatory light chain) non-covalently associated with it. The velocity of displacement increases with increasing numbers of active cross bridges up to a saturation level, that is, maximum unloaded sliding velocity. ${ }^{14}$ Based on a simple lever arm model, actin filament velocity is directly dependent on the length of the stalk. A genetically engineered truncated myosin with a $50 \%$ shorter lever arm moves actin at half its normal velocity." This is due to a reduction of the displacement distance to half by the shortened myosin stalk. Almost complcte omission of the lever arm (motor domain without light chain binding region) moves actin at less than $10 \%$ of its original speed. ${ }^{13}$ In both cases of truncated myosins the actin activated ATPase activity remained unchanged.

Nanomanipulations with a single actin filament attached to an ultracompliant glass microneedle makes it possible to measure force development by a single or very few myosin cross bridges. ${ }^{15}$ Recent results from such experiments show that the force production per single cross bridge and the ability to resist motion of different myosins can be ranked in the following order: smooth (activated by phosphorylation $)>$ fast skeletal $=\mathrm{V} 3$ cardiac $(\beta \beta$ myosin heavy chain homodimer) $>$ V1 cardiac ( $\alpha \alpha$ homodimer). ${ }^{16}$ The inverse ranking holds for three of these myosins with regard to their actin activated ATPase activities: V1 cardiac $>$ V3 cardiac $>$ smooth (phosphorylated). This indicates that myosins with low enzymatic activity work with a better economy than those with high enzymatic activity. The exception to the rule makes fast skeletal muscle myosin. This myosin may display some distinct properties which enable fast skeletal muscle to respond to a wider range of mechanical demands than smooth and cardiac muscle.

In the contraction mechanism with the myosin cross bridges as independent force generators actin is providing the track and enforces the directionality upon their movement. On the other hand, velocity of shortening, translocation distance per power stroke, and force development are all governed by the properties of the myosin motor. The unloaded sliding velocity is intimately coupled with the ATPase activity of a particular myosin isoform. The enzyme kinetics control the sequence of conformational changes associated with the attachment and detachment rates. The actin filament cannot move faster than the myosin heads can let go. Yet the translocation distance per power stroke of a cross bridge, or the step size, has recently come under debate. Under some circumstances the step size seems to be far in excess of the generally accepted $10 \mathrm{~nm} .{ }^{17}$ The puzzle may get resolved elegantly, again, by the new in vitro nanomanipulation techniques. A step size of around $110 \mathrm{~nm}$ was deduced for high sliding velocity $(70 \%$ of maximum of $9 \mu \mathrm{m}$ per second). At $20 \%$ of maximum velocity the step size was around $40 \mathrm{~nm}$, and under near isometric conditions the usual $8-10 \mathrm{~nm}$ step size is observed. Analysis of the force fluctuations indicated that the force must be produced by stochastic and independent attachment-detachment cycles between actin and myosin heads. It follows from this that multiple force generating attachment-detachment cycles correspond to a single ATPase cycle during sliding velocities $>20 \%$ of the maximum. With little sliding and during isometric contraction a displacement distance of one power stroke cycle is tightly coupled to hydrolysis of one ATP. The coupling between the ATPase and force generation cycles is not rigidly determined on a one to one fashion but is variable depending on the load. ${ }^{17}$

Taken together these new findings show that shortening velocity, translocation distance per power stroke, and force development depend on the enzyme kinetics of the various myosin isoforms. Some of the isoform specific properties can even be pinned down to a confined amino acid stretch of the myosin heavy chain in the motor domain. ${ }^{9}$ In addition, the 
mechanical load, varying from low (isotonic contraction) to high (isometric contraction), affects the cross bridge kinetics profoundly. Significantly more cross bridges are in the force producing state at any given time during isometric conditions.

Actin also exists in tissue specific isoforms. Its amino acid sequence is extremely conserved. Nevertheless, specific functional diversity may be expected, in particular between muscular and non-muscular actin isoforms. ${ }^{7}$ Two muscle actin isoforms (smooth and fast skeletal) have been shown in the in vitro motility assay to be translocated by smooth muscle myosin at the same rate. ${ }^{18}$ On the other hand, average force production per cross bridge of smooth and fast skeletal muscle myosin on the same actin filament is $0.6 \mathrm{pN}$ and 0.2 $\mathrm{pN}$, respectively. ${ }^{19}$ It seems therefore very unlikely that the large differences in contractile function reside in the actin filament instead of the myosin.

We have seen above that with increasing load a larger number of force producing cross bridges are attached to actin in the classic model. In the actin based motor model of Pollack,' the amount of force must be proportional to the amount of actin monomers which transform from "long" to "short". Under isometric conditions only the actin monomers in the non-overlap zone would be able to contribute to force production. At zero length change they would not actually shorten but would be kept under stress by the load. Actin monomers in the overlap cannot contribute, even if they were activated, since in the entire overlap zone the actin filament is held firmly in register by the attached myosin cross bridges. The number of attached cross bridges has no effect on isometric force production in this model.

Using a feedback enhanced laser trap system allows direct measurement of force and displacement distance of a single myosin molecule interacting with an actin filament. ${ }^{20}$ The actin filament is kept straight by fixation at its ends with two laser traps. The myosin is attached to a silica bead, which in turn is fixed onto a coverslip underneath. Discrete stepwise movements averaging $11 \mathrm{~nm}$ were detected under conditions of low load. Single force transients of 3-4 pN were measured under isometric conditions. These results represent new and convincing support for the classic swinging cross bridge model of muscle contraction.

How would now an actin based motor behave in the laser trap arrangement? The minimum requirement for actin filament shortening is two points of fixation, whether they be two laser traps or a $\mathrm{Z}$ line and a myosin crossbridge. If an isolated actin filament can be activated it should start oscillating between "long" and "short". If myosin on a silica bead was added in addition, the actin filament should stop oscillating. Instead, the shortening wave of the actin filament would approach the myosin cross bridge from the activated side. The cross bridge should detach and reattach after the lengthening wave has followed (see under description of actin based motor). As a consequence the actin filament should be displaced to one side under zero load condition. In this device the myosin serves only as a reversible fixation point directing the actin displacement onto one side. In fact, the result would look similar to what was found in the real experiment with an active cross bridge.

After having gone through many arguments for and against we realise that it is not easy to refute an actin based motor theory out of hand. It has to be mentioned, however, that the theory is not well documented so far and therefore not yet described in sufficient detail. On the other hand, enormous forays in new technologies in the past few years have led to a better understanding of the contractile mechanism. There is no doubt that most of the new insights are fully compatible with, and lend support to, the swinging cross bridge model. There is no urge for a shift of paradigm at present.

The ability to measure force and displacement of single motor molecules on the piconewton and nanometre level, respectively, represents a large step forward in exploring how chemical energy from ATP is transformed into mechanical work. The integration of these mechanical approaches with molecular genetics and structural analyses opens up a new epoch in muscle research.

We are grateful for support from the Swiss National Science Foundation, grant No 31-40694.94.

M C SCHAUB $\mathrm{D} \mathrm{KOCH}$

Institute of Pharmacology, University of Zurich, Zurich, Switzerland.

1 Pollack GH. Muscle-contraction mechanism: are alternative engines gathering steam? Cardiovasc Res 1995;29:737-46.

2 Eisenberg E, Hill TL. Muscle contraction and free energy transduction in biological systems. Science 1985:227:999-1006.

3 Huxley AF, Niedergerke R. Structural changes in muscle during contraction. Interference microscopy of living muscle fibres Nature 1954;173:971-3.

4 Huxley HE, Hanson J. Changes in the cross-striations of muscle during contraction and stretch and their structural interpretation Nature 1954;173:973-6.

5 Huxley HE. The mechanism of muscular contraction. Science 1969;164:1356-66.

6 Cold Spring Harbor Symposium. The mechanism of muscle contraction. Quant Biol 1973;37:1-706.

7 Sheterline P, Sparrow JC. Actin. Protein profile, vol 1, London: Academic Press, 1994:1-121.

8 Schutt CE, Lindberg U. Actin as the generator of tension during muscle contraction. Proc Natl Acad Sci USA 1992;89:319-23.

9 Spudich JA. How molecular motors work. Nature 1994;372: 515-8.

10 Rayment I, Rypniewski WR, Schmidt-Bäse K, et al. Threedimensional structure of myosin subfragment-1: a molecular motor. Science 1993;261:50-8.

11 Kabsch W, Mannherz HG, Suck D, Pai EF, Holmes KC. Atomic structure of the actin: DNAse I complex. Nature 1990;347: $37-48$.

12 Rayment I, Holden HM, Whittaker M, et al. Structure of the actinmyosin complex and its implications for muscle contraction. Science 1993;261:58-65.

13 Itakura S, Yamakawa H, Toyoshima YY, et al. Force-generating domain of myosin motor. Biochem Biophys Res Commun 1993;196:1504-10.

14 VanBuren P, Waller GS, Harris DE, Trybus KM, Warshaw DM, Lowey $S$. The essential light chain is required for full force production by skeletal muscle myosin. Proc Natl Acad Sci USA 1994:91:12403-7.

15 Yanagida T, Harada Y, Ishijima A. Nano-manipulation of actomyosin molecular motors in vitro: a new working principle. Trends Biochem Sci 1993;18:319-24

16 Harris DE, Work SS, Wright RK, Alpert NR, Warshaw DM Smooth, cardiac and skeletal muscle myosin force and motion generation assessed by cross-bridge mechanical interactions in vitro. J Muscle Res Cell Motil 1994;15:11-19.

17 Yanagida T, Ishijima A, Saito K, Harada Y. Coupling between ATPase and force-generating attachment-detachment cycles of actomyosin in vitro. Adv Exp Med Biol 1993;332:339-47.

18 Umemoto S, Sellers JR. Characterization of in vitro assays using smooth muscle and cytoplasmic myosins. J Biol Chem 1990; 265:14864-9.

19 VanBuren P, Work SS, Warshaw DM. Enhanced force generation by smooth muscle myosin in vitro. Proc Natl Acad Sci USA 1994;91:202 -5

20 Finer TJ, Simmons RM, Spudich JA. Single myosin molecule mechanics: piconewton forces and nanometre steps. Nature 1994;368:113-9. 